\title{
Distributed Leader-following Consensus of Nonlinear Multi-agent Systems with Nonlinear Input Dynamics
}

\author{
Yi Shi ${ }^{a}$, Yanyan Yin ${ }^{b}$, Song Wang ${ }^{b}$, Yanqing Liu ${ }^{a}$, Fei Liu ${ }^{a}$ \\ ${ }^{a}$ Key Laboratory of Advanced Process Control for Light Industry (Ministry of Education), \\ Institute of Automation, Jiangnan University, Wuxi, 214122, China. \\ ${ }^{b}$ Department of Mathematics and Statistics, Curtin University, GPO Box U1987, Perth WA 6845, Australia.
}

\begin{abstract}
This paper addresses the problem of distributed leader-following consensus for a multi-agent system with an affine nonlinear term. The communication topology we adopt is an undirected connected graph and the leader sends its information to one or more followers. To make each follower asymptotically synchronize with the leader, a nonlinear distributed control protocol is proposed. Using a Lyapunov function and a matrix theory, we establish sufficient conditions which ensure the consensus of these nonlinear multi-agent systems. Finally, a numerical simulation is provided to verify the effectiveness and usefulness of the developed method.
\end{abstract}

Keywords: Multi-agent systems, Leader-following consensus, Distributed control, Nonlinear systems.

\section{Introduction}

Inspired by natural biological consensus behaviors, considerable attention has been extensively paid to the consensus of agents by virtue of its widespread range of applications in many areas, such as robots, sensor networks, biological systems, formation and cooperative control, just to name a few [1-6]. As the most essential and important problems, consensus problems for multi-agent systems have drawn attention greatly in recent years [7-10]. From first-order systems [7], linear systems [8] to high-order systems [9], and then, nonlinear systems [10], the researches on consensus control are getting deeper. The consensus is considered to be achieved if each agent in the network converges to a certain common value. A principal problem for the consensus control is to construct effective control algorithms and laws based on the neighbor information to make some agents reach consensus under the corresponding topology.

In general, the existing results about consensus control concentrate on two types of control problems, namely leaderless consensus and leader-following consensus. The former requests that each agent converges to a certain agreement state [11-13], which is related to the initial conditions. For example, the consensus problems for a network of first-order agents are addressed in [11]. Each agent eventually converges to the average of the initial 
states under balanced digraphs. Recently, consensus of multi-agent systems with a leader has been extensively studied [14-17], whose goal is to guarantee that a group of agents can track the state trajectory of a leader. In [14], the authors concern the consensus for first-order networks with a time-varying leader. The study in [15] is an extension of [14] from first-order to high-order systems. In [16], the semi-global leader-following consensus is investigated for linear systems, whose actuators are imperfect. In [17], global leader-following consensus is discussed under bounded controls. It is true that with the help of a leader, the application range is enlarged for multi-agent systems.

Till now, many results on simple linear multi-agent systems can be found in the open literature, including most references mentioned above. However, in practice, most of engineering problems involve complex nonlinear systems so that results for linear systems do not apply. Hence, the nonlinearities in dynamics have been taken into consideration for many researchers recently. In [18], the authors concern the consensus of third-order multi-agent systems with nonlinear dynamics under a fixed topology. In [19], tracking consensus is studied for nonlinear multi-agent systems. As an extension, the switching networks and unreliable communication are further discussed in [20].

Compared with results in $[19,20]$, the affine nonlinear term is further introduced for the nonlinear systems in this paper. There is no doubt that affine nonlinear systems can simulate many actual systems in many fields, including mechanics, physics, ecology, and engineering [21-23]. The stability analysis of affine nonlinear systems is given in [24]. To the best of our knowledge, very few results on consensus problem of affine nonlinear systems can be found in the open literature. For example, the consensus problem is addressed for nonlinear multi-agent systems in [25], which are affine in control. However, there is no research on the tracking consensus for affine nonlinear systems. Based on the discussion above, a type of nonlinear systems with the affine nonlinear term are proposed and the leader-following consensus is introduced to them in this work. Motivated by our previous works on nonlinear controllers design for nonlinear systems [26-27], we develop a numerical method for leaderfollowing problem involving a type of affine nonlinear systems. More specifically, we construct a novel nonlinear control algorithm for each follower agent, under which sufficient conditions are obtained for reaching consensus. We assume that the followers communicate with the undirected and connected graph, and the leader sends its message to one or more followers. The key contributions of the paper are threefold. (1) Compared with the existing conclusions, this work can handle a class of more general nonlinear systems with affine nonlinear term. (2) The tracking consensus is investigated for the nonlinear systems. (3) The relationship between design parameters and consensus performance has been built and revealed to guarantee the consensus.

The overall structure of the rest of the paper is as follows. A problem statement and preliminaries, which include some definitions, assumptions and some other basic graph knowledge, are illustrated in Section 2. In Section 3, we design a nonlinear control protocol for each follower, and sufficient conditions are provided to ensure the consensus of the system proposed. In Section 4, we present some simulation results to demonstrate the effectiveness and usefulness of our method by using a non-trivial test example. Section 5 makes a brief summary finally.

Notation. The following notations are applied throughout the paper. For given positive integers $m$ and $n, R^{n}$ stands for the Euclidean space of $n$ dimensional, and $R^{m \times n}$ represents a set of $m \times n$ matrices. We use $1_{N}$ to denote the a column vector in $R^{N}$ whose entries are all equal to one. $I_{N}$ means the $N \times N$ identity matrix. $\|\cdot\|$ is the Euclidean norm of a certain vector. If not specifically pointed out, the dimensions of each matrix is supposed to be appropriate. Furthermore, we use $\operatorname{diag}\{\cdot\}$ to denote a matrix, whose diagonal are the corresponding parameter values, and other entries are all zero. The transpose of a matrix $A$ is symbolled by $A^{T}$ and $\otimes$ is the Kronecker product, which has many properties, such as $(A \otimes B)(C \otimes D)=A C \otimes B D$ and $(A \otimes B)^{T}=A^{T} \otimes B^{T}$. For a square matrix $P, P>0$ or $P<0$ represents it is a positive or negative definite 
matrix respectively.

\section{Problem Statement and Preliminaries}

In the section, we give a brief account of some graph theory basis and present the leader-following consensus problem.

\subsection{Graph Theory}

Graphs are often applied to model the communication rules among the agents. Take a graph $G=\{v, \varepsilon, A\}$ with $N$ nodes as example, $N$ nodes (follower agents) are represented by $v=\left\{v_{1}, v_{2}, \ldots, v_{N}\right\}$, and the leader is labeled as $v_{0}$. All edges or arcs are denoted as $\varepsilon \subseteq v \times v$. The pair $\left(v_{j}, v_{i}\right) \in \varepsilon$ means there is an edge connecting the agents $j$ and $i$. Agent $j$ is known as a neighbor of agent $i$ if $\left(v_{j}, v_{i}\right) \in \varepsilon$. The structure of $G$ can also be described by the adjacency matrix $A=\left[a_{i j}\right]$. If agent $i$ can get information from agent $j, a_{i j}>0$, otherwise $a_{i j}=0$. We suppose that $a_{i i}=0$ for all $i=1,2, \ldots, N$. The in-degree of agent $i$ is denoted as

$d_{i}=\sum_{j=1}^{N} a_{i j}$, and the in-degree matrix $D$ is a diagonal matrix with $D=\operatorname{diag}\left\{d_{i}\right\} \in R^{N \times N}$. Let Laplacian matrix be $L=D-A$. If the leader can send its information to agent $i, a_{i 0}>0$ for $i=1,2, \ldots, N$, otherwise $a_{i 0}=0$. The graph is said to be undirected if $a_{i j}=a_{j i}$, i.e., the agent $i$ and $j$ can communicate with each other. If there is an undirected path between any two vertices, the undirected graph is called connected. We put $M=L+\operatorname{diag}\left\{a_{10}, a_{20}, \cdots, a_{N 0}\right\}$.

\subsection{Problem Statement}

Consider $N$ communicated agents with the dynamics of them described as,

$$
\dot{x}_{i}=A x_{i}+B f\left(x_{i}\right)+g\left(x_{i}\right) u_{i}
$$

where $x_{i} \in R^{n}$ is the state vector of agent $i, i=1,2, \ldots N$, and $u_{i} \in R^{m}$ is the control input. $f\left(x_{i}(t)\right) \in R^{r}$ and $g\left(x_{i}\right)=\left[g_{1}\left(x_{i}\right), g_{2}\left(x_{i}\right), \ldots, g_{m}\left(x_{i}\right)\right] \in R^{n \times m}$ mean the intrinsic nonlinear dynamics. $A \in R^{n \times n}$ and $B \in R^{n \times r}$ are both constant system matrices.

The dynamics of the leader is described by the following nonlinear form,

$$
\dot{x}_{0}=A x_{0}+B f\left(x_{0}\right)
$$

where $x_{0} \in R^{n}$ is the state of the leader. Its state trajectory evolves without being effected by the followers, and the leader provides the information for the followers to track its trajectory.

Definition 1 [28] For any any initial condition $x_{i}(0), i=0,1,2, \cdots, N$, the leader-following consensus of the system (1)-(2) is achieved if there exists a control input $u_{i}$ that makes the system satisfy the following relationship:

$$
\lim _{t \rightarrow \infty}\left\|x_{i}(t)-x_{0}(t)\right\|=0, i=1,2, \cdots, N
$$

The communication topology connecting the agents is considered to satisfy the assumption as below. 
Assumption 1 The undirected topology $G$ we adopt is connected, and the leader sends its information to one or more followers.

Remark 1 Some literature have investigated the consensus with directed connected graphs, such as [29, 30] for continuous-time systems, and [31] for discrete-time systems. In the future work, more general communication networks will be taken into consideration.

Assumption 2 For a constant $\rho>0$, the nonlinear dynamics $f(x)$ satisfies the Lipschitz condition as follows,

$$
\|f(a)-f(b)\| \leq \rho\|a-b\|, \forall a, b \in R^{n} .
$$

Assumption 3 For constant system matrix A, there exists a positive matrix $P>0$, which satisfies the equation $A^{T} P+P A=Q$ for some $Q<0$.

Remark 2 If Assumption 3 is satisfied, an appropriate positive matrix $P$ can be obtained by condition (4).

\section{Main Results}

In the section, we design the following distributed control protocol for each follower,

$$
u_{i}(t)=-g^{T}\left(x_{i}(t)\right) P\left(\sum_{j=1}^{N} a_{i j}\left(x_{i}-x_{j}\right)+a_{i 0}\left(x_{i}-x_{0}\right)\right),
$$

in which $a_{i j}$, the elements of matrix $A$, means the communication weight among the followers, $a_{i 0}$ is the weight between the leader and each follower agent, and $P$ is a positive matrix.

Theorem 1 Consider the followers and the leader whose dynamics are respectively described by (1) and (2). Suppose that Assumptions 1, 2 and 3 hold. The consensus of the system (1)-(2) is achieved under the protocol (3) if there exists a positive matrix $P$ that makes the following condition hold:

$$
P A+A^{T} P+2 \rho \sigma_{m}(B) P<0,
$$

where $\sigma_{m}(B)$ is the maximum singular value of constant matrix $B$.

Proof. Let

$$
\begin{aligned}
& x(t)=\left[x_{1}^{T}(t), \cdots, x_{N}^{T}(t)\right] \in R^{n N}, \\
& F(x(t))=\left[f\left(x_{1}(t)\right)^{T}, \cdots, f\left(x_{N}(t)\right)^{T}\right]^{T} \in R^{r N}, \\
& u=\left[u_{1}^{T}, u_{2}^{T}, \ldots, u_{N}^{T}\right]^{T} \in R^{m N}, \\
& G(x)=\left[\begin{array}{cccc}
g\left(x_{1}\right) & & & \\
& g\left(x_{2}\right) & & \\
& & \ddots & \\
& & & g\left(x_{N}\right)
\end{array}\right] \in R^{N n \times N m},
\end{aligned}
$$


then the dynamics of the followers are rewritten as

$$
\dot{x}(t)=\left(I_{N} \otimes A\right) x(t)+\left(I_{N} \otimes B\right) F(x(t))+G(x) u(t),
$$

and the control input (3) gives

$$
u(t)=-G^{T}(x(t))(M \otimes P) d(t),
$$

where $M=L+\operatorname{diag}\left\{a_{10}, a_{20}, \cdots, a_{N 0}\right\}$.

The tracking error is denote as $d_{i}(t)=x_{i}(t)-x_{0}(t)$. then we have

$$
\dot{d}_{i}(t)=A d_{i}(t)+B\left(f\left(x_{i}(t)\right)-f\left(x_{0}(t)\right)\right)+g\left(x_{i}(t)\right) u_{i}(t) .
$$

Let $d(t)=\left[d_{1}^{T}(t), d_{2}^{T}(t), \cdots, d_{N}^{T}(t)\right]^{T} \in R^{n N}$. The error dynamic is continue as

$$
\begin{aligned}
\dot{d}(t) & =\dot{x}(t)-\dot{x}_{0}^{\prime}(t) \\
& =\left(I_{N} \otimes A\right)\left(x(t)-x_{0}^{\prime}(t)\right)+\left(I_{N} \otimes B\right)\left(F(x(t))-f^{\prime}\left(x_{0}(t)\right)\right)+G(x) u(t) \\
& =\left(I_{N} \otimes A\right) d(t)+\left(I_{N} \otimes B\right)\left(F(x(t))-f^{\prime}\left(x_{0}(t)\right)\right)+G(x) u(t),
\end{aligned}
$$

where $x_{0}^{\prime}=x_{0} 1_{N}$ and $f^{\prime}\left(x_{0}\right)=f\left(x_{0}\right) 1_{N}$.

Define $F^{\prime}(x(t))=F(x(t))-f^{\prime}\left(x_{0}(t)\right)$. Substituting (6) into (8), we have

$$
\begin{aligned}
\dot{d}(t)= & \left(I_{N} \otimes A\right) d(t)+\left(I_{N} \otimes B\right) F^{\prime}(x(t)) \\
& +G(x)\left(-G^{T}(x(t))(M \otimes P) d(t)\right) .
\end{aligned}
$$

Choose the Lyapunov function as follows,

$$
V(t)=d^{T}(t)(M \otimes P) d(t)
$$

where $M>0$ and $P>0$.

Differentiating $V(t)$ yields,

$$
\begin{aligned}
\dot{V}(t)= & \dot{d}^{T}(t)(M \otimes P) d(t)+d^{T}(t)(M \otimes P) \dot{d}(t) \\
= & d^{T}(t)(M \otimes P A) d(t)+d^{T}(t)\left(M \otimes A^{T} P\right) d(t) \\
& +2 d^{T}(t)(M \otimes P)\left(I_{N} \otimes B\right) F^{\prime}(x(t)) \\
& +2 d^{T}(t)(M \otimes P) G(x)\left(-G^{T}(x)(M \otimes P) d(t)\right) \\
= & d^{T}(t)\left(M \otimes\left(P A+A^{T} P\right)\right) d(t) \\
& +2 d^{T}(t)(M \otimes P B) F^{\prime}(x(t)) \\
& -2 d^{T}(t)(M \otimes P) G(x)\left(G^{T}(x)(M \otimes P) d(t)\right) .
\end{aligned}
$$


Let $\dot{V}(t)=\dot{V}_{1}(t)+\dot{V}_{2}(t)$, where

$$
\begin{gathered}
\dot{V}_{1}(t)=d^{T}(t)\left(M \otimes P A+A^{T} P\right) d(t) \\
+2 d^{T}(t)(M \otimes P B) F^{\prime}(x(t)), \\
\dot{V}_{2}(t)=-2 d^{T}(t)(M \otimes P) G(x)\left(G^{T}(x)(M \otimes P) d(t)\right) .
\end{gathered}
$$

Since both matrices $P$ and $M$ are positive definite, $\dot{V}_{2}(t) \leq 0$.

From Assumption 1, we have $M>0$. Then, there must be an orthogonal matrix $S \in R^{N \times N}$ such that

$$
M=S^{T} \operatorname{diag}\left\{\lambda_{1}, \lambda_{2}, \cdots, \lambda_{N}\right\} S .
$$

For brevity, let $\Lambda=\operatorname{diag}\left\{\lambda_{1}, \lambda_{2}, \cdots, \lambda_{N}\right\}$.

Using Assumption 2, we can estimate the second term in (10) as follows,

$$
\begin{aligned}
& 2 d^{T}(t)(M \otimes P B) F^{\prime}(x(t)) \\
= & 2 d^{T}(t)\left(S^{T} \Lambda S \otimes P\right)\left(I_{N} \otimes B\right) F^{\prime}(x(t)) \\
= & 2 d^{T}(t)\left(S^{T} \Lambda S \otimes P\right)\left(I_{N} \otimes B\right)\left(F(x(t))-f\left(x_{0}\right) 1_{N}\right) \\
\leq & 2 \rho \sigma_{m}(B) d^{T}(t)(M \otimes P) d(t) .
\end{aligned}
$$

Substituting (12) into (10), we obtain

$$
\begin{aligned}
\dot{V}_{1}(t) & =d^{T}(t)\left(M \otimes\left(P A+A^{T} P\right)\right) d(t)+2 \rho \sigma_{m}(B) d^{T}(t)(M \otimes P) d(t) \\
& =d^{T}(t)\left(M \otimes\left(P A+A^{T} P+2 \rho \sigma_{m}(B) P\right)\right) d(t) .
\end{aligned}
$$

Since $M=S^{T} \Lambda S$, then (13) is given as

$$
\dot{V}_{1}(t)=d^{T}(t)\left(S^{T} \Lambda S \otimes\left(P A+A^{T} P+2 \rho \sigma_{m}(B) P\right)\right) d(t) .
$$

Let $\tilde{d}(t)=\left[\tilde{d}_{1}^{T}(t), \tilde{d}_{2}^{T}(t), \cdots, \tilde{d}_{N}^{T}(t)\right]^{T}=\left(S \otimes I_{n}\right) d(t) . \dot{V}_{1}(t)$ is rewritten as

$$
\begin{aligned}
\dot{V}_{1}(t) & =\tilde{d}^{T}(t)\left(\Lambda \otimes\left(P A+A^{T} P+2 \rho \sigma_{m}(B) P\right)\right) \tilde{d} \\
& =\sum_{i=1}^{N} \lambda_{i} \tilde{d}_{i}^{T}\left(P A+A^{T} P+2 \rho \sigma_{m}(B) P\right) \tilde{d}_{i} .
\end{aligned}
$$

From condition (4), we have $\dot{V}_{1}(t)<0$. We can easily find that $\dot{V}(t)<0$. Thus, $\lim _{t \rightarrow \infty} d(t)=0$. This completes the proof.

Remark 3 Under condition (4) in Theorem 1, the consensus is reached asymptotically with the protocol (3). In the future work, consensus achieved globally exponentially and the improvement to the convergence rate will be taken into consideration. 


\section{Simulation Results}

In our simulation, the multi-agent network composed of a leader and five followers is considered and shown in Figure 1.

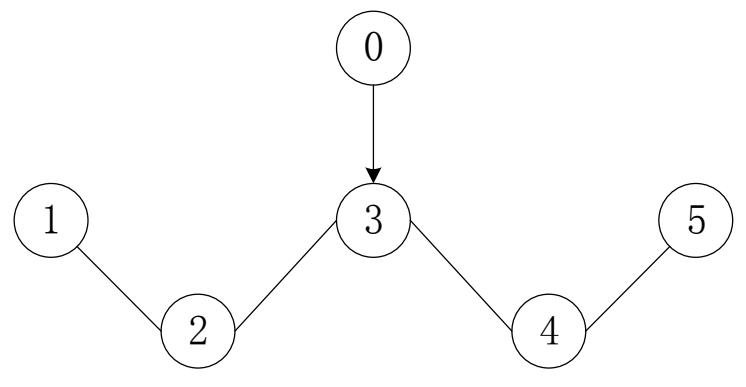

Figure 1: The undirected graph consisting of five follower nodes and a leader node

The dynamics of the systems, which include a leader and five followers, are respectively given as,

$$
\begin{gathered}
\dot{x}_{0}=A x_{0}+B f\left(x_{0}\right), \\
\dot{x}_{i}=A x_{i}+B f\left(x_{i}\right)+g\left(x_{i}\right) u_{i},
\end{gathered}
$$

where $x_{i} \in R^{2}, u_{i} \in R, i=1,2, \cdots, 5$,

$$
\begin{gathered}
A=\left[\begin{array}{rr}
9 & -15 \\
18 & -20
\end{array}\right], B=\left[\begin{array}{l}
1 \\
0
\end{array}\right], \\
g\left(x_{i}\right)=\left[\begin{array}{c}
x_{i 2} \\
x_{i 1}^{2}+3 x_{i 2}^{2}
\end{array}\right] .
\end{gathered}
$$

We choose $f\left(x_{i}(t)\right)=k_{2} x_{i 1}(t)+0.5\left(k_{1}-k_{2}\right)\left(\left|x_{i 1}(t)+1\right|-\left|x_{i 1}(t)-1\right|\right)$, in which $k_{1}=-1.31$ and $k_{2}=$ 0.75. From [32,33] and Assumption 2, we have $\rho=1.31$, and Assumption 3 holds as well.

Applying Theorem 1, the corresponding feasible solution of matrix $P$ is found to be

$$
P=\left[\begin{array}{cc}
0.6998 & -0.4328 \\
-0.4328 & 0.3785
\end{array}\right]
$$


In the simulation part, we set the initial states of these six agents as

$$
\begin{aligned}
& {\left[x_{0}(0), x_{1}(0), x_{2}(0), x_{3}(0), x_{4}(0), x_{5}(0)\right]} \\
& =\left[\begin{array}{cccccc}
0.5 & 1.2 & -0.75 & -1.85 & 1.5 & 0.8 \\
-1 & 0.8 & 1.5 & -0.5 & -1.3 & 0.1
\end{array}\right]
\end{aligned}
$$

and suppose that the graph in Figure 1 has $0-1$ communication weights.

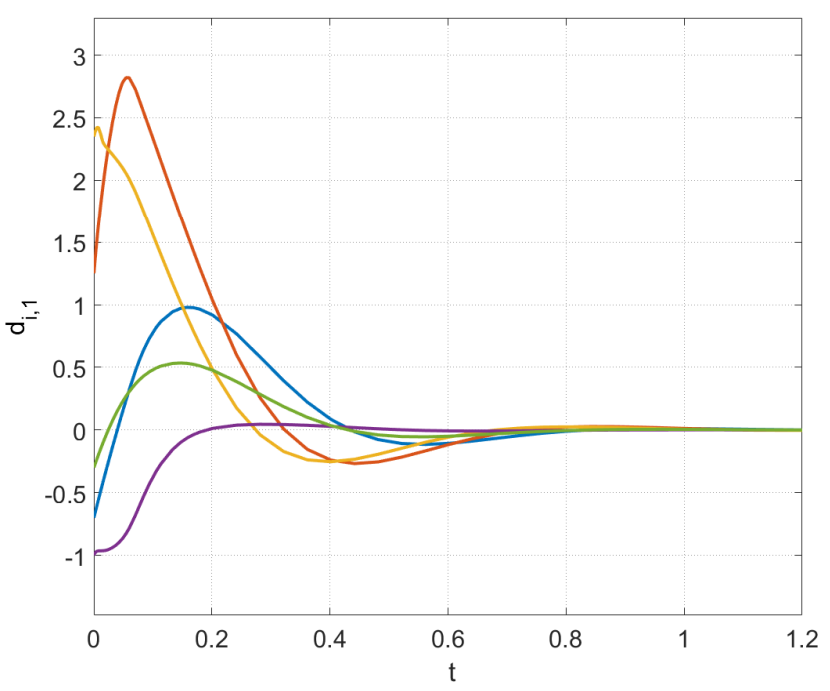

Figure 2: Trajectories of state tracking errors $d_{i, 1}$

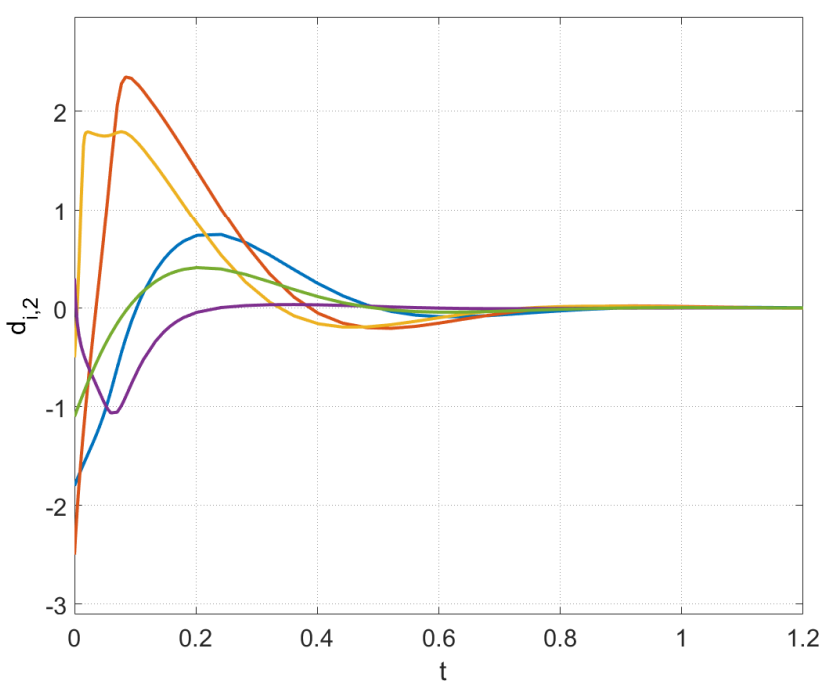

Figure 3: Trajectories of state tracking errors $d_{i, 2}$

The simulation images are demonstrated in Figures 2 and 3, from which we know that the leader is followed by the five agents asymptotically, i.e., the consensus is observed. 


\section{Conclusions}

In this work, the distributed leader-following consensus is introduced and investigated for a type of multiagent systems with some nonlinear dynamics under undirected topology. Under the nonlinear control algorithm proposed, a numerical example is provided to show the correctness of the theoretical method. Future work will include consensus with some more complex situations, such as nonlinear systems with stochastic parameters, communication delays, or more general communication topologies, etc.

\section{Acknowledgment}

This work was supported in part by the Ministry of Education of China under the 111 Project B12018, the National Natural Science Foundation of China under grant No. 61403169, 61773011, and Curtin Fellowship.

\section{References}

[1] R. Vidal, O. Shakernia, and S. Sastry, Formation control of nonholonomic mobile robots with omnidirectional visual servoing and motion segmentation, IEEE International Conference on Robotics and Automation, 2003, 11(4), 14-20.

[2] J. Feddema, C. Lewis, and D. Schoenwald, Decentralized control of cooperative robotic vehicles: theory and application, IEEE Transactions on Robotics \& Automation, 2002, 18(5), 852-864.

[3] H. Dong, Z. Wang, and H. Gao, Distributed Filtering for a Class of Time-Varying Systems Over Sensor Networks With Quantization Errors and Successive Packet Dropouts, IEEE Transactions on Signal Processing, 2012, 60(6), 3164-3173.

[4] J. A. Fax and R. M. Murray, Information flow and cooperative control of vehicle formations, IEEE Transactions on Automatic Control, 2002, 35(1), 115-120.

[5] R. Olfati-Saber, Flocking for multi-agent dynamic systems, Algorithms and Theory, IEEE Transactions on Automatic Control, 2006, 51(3), 401-420.

[6] W. Ren, R. W. Beard, and E. M. Atkins, Information consensus in multivehicle cooperative control, IEEE Control Systems Magazine, 2007, 27(2), 71-82.

[7] W. Ren, and R. W. Beard, Consensus seeking in multiagent systems under dynamically changing interaction topologies, IEEE Transactions on automatic control, 2005, 50(5), 655-661.

[8] T. Xie, X. Liao, and H. Li, Leader-following consensus in second-order multi-agent systems with input time delay: An event-triggered sampling approach, Neurocomputing, 2016, 177, 130-135.

[9] B. Hou, F. Sun, H. Li, Y. Chen, and G. Liu, Observer-based cluster consensus control of high-order multiagent systems, Neurocomputing, 2015, 168, 979-982.

[10] J. Liu, Z. Liu, and Z. Chen, Coordinative control of multi-agent systems using distributed nonlinear output regulation, Nonlinear Dynamics, 2012, 67(3), 1871-1881.

[11] R. Olfati-Saber and R. M. Murray, Consensus problems in networks of agents with switching topology and time-delays, IEEE Transactions on Automatic Control, 2004, 49(9), 1520-1533. 
[12] W. Ren, On consensus algorithms for double-integrator dynamics, IEEE Transactions on Automatic Control, 2008, 53(6), 1503-1509.

[13] Y. Pei and J. Sun, Consensus of discrete-time linear multi-agent systems with Markov switching topologies and time-delay, Neurocomputing, 2015, 151(2), 776-781.

[14] W. Ren, Multi-vehicle consensus with a time-varying reference state, Systems \& Control Letters, 2007, $56(7), 474-483$.

[15] W. Ren, K. L. Moore, and Y. Q. Chen, High-order and model reference consensus algorithms in cooperative control of multivehicle systems, Journal of Dynamic Systems, Measurement, and Control, 2007, 129(5), 678-688.

[16] L. Shi, Z. Zhao, and Z. Lin, Robust semi-global leader-following practical consensus of a group of linear systems with imperfect actuators, Science China Information Sciences, 2017, 60(7), 072201.

[17] Z. Zhao and Z. Lin, Global leader-following consensus of a group of general linear systems using bounded controls, Automatica, 2016, 68, 294-304.

[18] Y. Xin, Y. Li, X. Huang, and Z. Cheng, Consensus of third-order nonlinear multi-agent systems, Neurocomputing, 2015, 159(1), 84-89.

[19] Y. Zhao, Z. Li, and Z. Duan, Distributed consensus tracking of multi-agent systems with nonlinear dynamics under a reference leader, International Journal of Control, 2013, 86(10), 1859-1869.

[20] B. Cui, C. Zhao, T. Ma, and C. Feng, Leader-following consensus of nonlinear multi-agent systems with switching topologies and unreliable communications, Neural Computing and Applications, 2016, 27(4), 909-915.

[21] S. El-Ferik, A. Qureshi, and F. L. Lewis, Neuro-adaptive cooperative tracking control of unknown higherorder affine nonlinear systems, Automatica, 2014, 50(3), 798-808.

[22] H. Zhang, Y. Luo, and D. Liu, Neural-network-based near-optimal control for a class of discrete-time affine nonlinear systems with control constraints, IEEE Transactions on Neural Networks, 2009, 20(9), 1490-1503.

[23] C. L. Hwang and C. Y. Kuo, A stable adaptive fuzzy sliding-mode control for affine nonlinear systems with application to four-bar linkage systems, IEEE Transactions on Fuzzy Systems, 2001, 9(2), 238-252.

[24] W. Lin, Input saturation and global stabilization of nonlinear systems via state and output feedback, IEEE Transactions on Automatic Control, 1995, 40(4), 776-782.

[25] H. Hu, S. Y. Yoon, and Z. Lin, Consensus of multi-agent systems with control-affine nonlinear dynamics, Unmanned Systems, 2016, 04(01), 61-73.

[26] Y. Yin, P. Shi, F. Liu, K. L. Teo, and C. C. Lim, Robust filtering for nonlinear nonhomogeneous Markov jump systems by fuzzy approximation approach, IEEE Transactions on Cybernetics, 2015, 45(9), 1706-1716.

[27] Y. Yin and Z. Lin, Constrained control of uncertain nonhomogeneous Markovian jump systems, International Journal of Robust and Nonlinear Control, 2017, DOI: 10.1002/rnc.3774.

[28] W. Zhu, M. Wang, and C. Yang, Leader-following consensus of fractional-order multi-agent systems with general linear models, Applied Mathematics \& Mechanics, 2015, 88(9), 1-11. 
[29] W. Yu, G. Chen, M. Cao, and J. Kurths, Second-order consensus for multiagent systems with directed topologies and nonlinear dynamics, IEEE Transactions on Systems, Man, and Cybernetics, Part B: Cybernetics, 2010, 40(3), 881-891.

[30] J. Lu, D. W. C. Ho, and J. Kurths, Consensus over directed static networks with arbitrary finite communication delays, Physical Review E, 2009, 80(6), 066121.

[31] Y. Liu, D. W. C. Ho, and Z. Wang, A new framework for consensus for discrete-time directed networks of multi-agents with distributed delays, International Journal of Control, 2012, 85(11), 1755-1765.

[32] H. Agiza and A. Matouk, Adaptive synchronization of Chuas circuits with fully unknown parameters, Chaos Solitons \& Fractals, 2006, 28(1), 219-227.

[33] Z. Li, C. Wen, Y. Soh, and W. Xie, The stabilization and synchronization of Chua's oscillators via impulsive control, IEEE Transactions on Circuits \& Systems I Fundamental Theory \& Applications , 2001, 48(11), $1351-1355$. 